\title{
Comparison of Repetitive Transcranial Magnetic Stimulation and Transcranial Direct Current Stimulation on Upper Limb Recovery Among Patients With Recent Stroke
}

\author{
Ka Ying Doris Miu, MBBS, MRCP ${ }^{1}$, Ching Kok, MBChB, DPD ${ }^{1}$, Sau Shan Leung, RN, BHS ${ }^{1}$, \\ Elaine Y. L. Chan, OT, MSc ${ }^{2}$, Elaine Wong, PT, $\mathrm{MSc}^{3}$
}

Departments of ${ }^{1}$ Rehabilitation and Extended Care, ${ }^{2}$ Occupational Therapy, and ${ }^{3}$ Physiotherapy, Wong Tai Sin Hospital, Hong Kong, China

\begin{abstract}
Objective To compare the efficacy of repetitive transcranial magnetic stimulation (rTMS) and transcranial direct current stimulation (tDCS) on upper limb function recovery among patients who recently had stroke.

Methods Subjects with recent stroke (within 1 month) were randomized to rTMS ( $\mathrm{n}=25)$ and tDCS ( $\mathrm{n}=26)$ applied over the non-lesioned hemisphere for three sessions per week, followed by tailored upper limb rehabilitation training for a total of 2 weeks. The primary outcomes were changes in the Motor Assessment Scale (MAS), FuglMeyer arm score test, Nine-Hole Peg Test (9HPT), hand grip strength, and modified Barthel Index at weeks 2 and 4. Both therapists responsible for training and assessment were blinded to the intervention allocated.

Results There was an improvement in all the motor performance scales among both groups $(\mathrm{p}<0.001)$. These improvements persisted at discharge. However, there was no significant difference in any of the assessment scales between the two groups. The rTMS group showed a statistically non-significant greater improvement in MAS, 9HPT, and handgrip strength than the tDCS group.

Conclusion Both interventions produce a statistically significant improvement in upper limb function. There was no statistically significant difference between the two intervention methods with respect to motor performance. It is suggested that a larger study may help to clarify the superiority of either methods.
\end{abstract}

Keywords Transcranial direct current stimulation (tDCS), Transcranial magnetic stimulation, rTMS, Stroke, Upper limb motor recovery

Received March 7, 2020; Revised July 21, 2020; Accepted September 10, 2020; Published online December 31, 2020

Corresponding author: Ka Ying Doris Miu

Department of Rehabilitation and Extended Care, Wong Tai Sin Hospital, 124 Shatin Pass Road, Hong Kong, China. Tel: $+852-35713668$, Fax: $+852-$ 35713855, E-mail: miuky@ha.org.hk

ORCID: Ka Ying Doris Miu (https://orcid.org/0000-0003-2923-4882); Ching Kok (https://orcid.org/0000-0003-0965-2016); Sau Shan Leung (https:// orcid.org/0000-0002-7882-2982); Elaine Y. L. Chan (https://orcid.org/0000-0002-1461-3591); Elaine Wong (https://orcid.org/0000-0002-6295-3267).

(c) This is an open-access article distributed under the terms of the Creative Commons Attribution Non-Commercial License (http://creativecommons.org/ licenses/by-nc/4.0) which permits unrestricted noncommercial use, distribution, and reproduction in any medium, provided the original work is properly cited. Copyright () 2020 by Korean Academy of Rehabilitation Medicine 


\section{INTRODUCTION}

Limitation of upper limb function is considered a disabling and disturbing consequence of stroke. Besides other functional disabilities, it can add to a negative effect on quality of life and social participation $[1,2]$. In a study of stroke subjects with a paretic upper limb, about $33 \%-66 \%$ of them were unable to recover arm function in the first 6 months after stroke [3]. Moreover, up to $50 \%$ of the patients did not show any sign of significant arm recovery after $>5$ years post-stroke [4]. It has been suggested in two systemic reviews $[5,6]$ that stroke rehabilitation intensity is an important factor associated with greater and faster recovery. On the other hand, due to resource limitations, there is an increasing need for effective treatment without adding the cost of the healthcare system. Studies have suggested that post-stroke functional improvement can be augmented by neuromodulatory intervention through non-invasive brain stimulation (NIBS), which involves transcranial magnetic stimulation and transcranial direct current stimulation (tDCS), together with physical training $[7,8]$. Repetitive transcranial magnetic stimulation (rTMS) is a non-invasive technology that aims at maximizing recovery of function after stroke. Repetitive delivery of TMS (rTMS) with highfrequency pulses will enhance excitability in the cortex, while repetitive low frequency rTMS suppresses cortical excitability $[9,10]$. There is an imbalance between the damaged and undamaged brain hemispheres in hemiplegic stroke patients, which was shown to have reduced excitability of the ipsilesional motor cortex and increased excitability in the contra-lesioned motor cortex [11]. Improvement in upper limb function after the application of low-frequency rTMS to the non-lesioned hemisphere has been proven in several studies [12,13]. Hence, rTMS can be used as an add-on treatment for routine rehabilitation programs in patients with acute stroke.

tDCS is another non-invasive method that can modulate neuronal excitability in a polarity-specific manner. It involves the application of a weak electrical direct current to stimulate the brain. Anodal tDCS of the primary motor cortex (M1) increases corticospinal excitability, while cathodal tDCS exerts an inhibitory effect $[14,15]$. Thus, tDCS can be used for the rehabilitation of motor function in patients with stroke and has been shown to have promising results [16].
Both rTMS and tDCS have shown their effectiveness in the recovery of upper limb function. rTMS involves more tedious manpower input and higher equipment cost, while tDCS is simpler and can be applied at bedside. At present, studies comparing the effects of these two NIBS methods are minimal. Identification of the most beneficial stimulation methods for cortical excitability can help to determine the best treatment option for motor function improvement among stroke patients.

This study aimed to compare the efficacy of rTMS and tDCS on upper limb function recovery among a group of patients receiving rehabilitation in a rehabilitation hospital.

\section{MATERIALS AND METHODS}

Patients with recent stroke aged $>18$ years admitted to the rehabilitation unit of an extended care hospital were recruited. All stroke diagnoses were ascertained by neuroimaging. The inclusion criteria were subjects with ischemic or hemorrhagic stroke who could follow commands. Only participants who could read, write, and communicate in Chinese were recruited. Exclusion criteria were those who had (1) motor, sensory, or global aphasia; (2) inability to reach and grasp a dowel at least once; (3) conditions contraindicated to rTMS/tDCS, such as known seizure and had taken drugs known to lower the seizure threshold; and (4) having an intracranial metallic implant. Patients with stroke onset of $>1$ month were also excluded. Information collected included basic demographics, side of hemiparesis, and date of stroke onset. Infarct location was categorized according to the Oxfordshire classification [17], and the etiology of ischemic stroke was defined by the Trial of Org 10172 in Acute Stroke Treatment criteria [18].

All patients provided written informed consent to participate in the study. Approval from KCC clinical research ethics committee, Hong Kong Hospital Authority was obtained (No. REC [KC/KE]-18-0149/FR-4).

This was a single-blind randomized trial. Patients were randomly assigned to either rTMS or tDCS by a sealed envelope in a 1:1 ratio. All of them underwent standard upper limb training after the non-invasive electrical brain stimulation. Because the patients themselves were not blinded to the intervention modality, only the physiotherapist and occupational therapist responsible for the 
training and assessment of motor function were blinded to the mode of brain stimulation.

The standard duration of stroke rehabilitation was 4 weeks for all patients.

Measurements of motor functions were assessed by a trained physiotherapist and an occupational therapist who were blinded to the treatment allocation. Assessment was done at baseline, at 2 weeks post intervention, and at 4 weeks. The Motor Assessment Scale (MAS) [19] was used to assess motor function. It comprised an assessment of 8 areas of motor function, and 1 item was associated with muscle tone. Each item was scored on a scale of 0-6. A higher score indicated a better upper limb function. The modified Ashworth Scale (AS) was used to assess spasticity [20]. The Fugl-Meyer arm score test (FM) is an index for evaluating motor function [21]. It comprised 33 tasks performed with the affected upper limb. The performance of each task was rated, and a higher rating represented a better performance. The Nine-Hole Peg Test (9HPT) [22] examined fine motor skills and was expressed as pegs per second. Grip strength was recorded using a standard dynamometer. The modified Barthel In$\operatorname{dex}(\mathrm{mBI})$ was used to measure dependency and physical function.

\section{Intervention}

rTMS

Single-pulse TMS was used to measure non-lesioned motor cortex excitability. Stimulation was administered using a PowerMAG 100 clinical stimulator (MAG and More GmbH, Munich, Germany) with air-cooled (figure-8) coil and evoked responses were measured from the abductor pollicis brevis (APB) on the non-paretic side. The "hot spot" was identified as the optimal scalp position for eliciting the largest motor evoked potential (MEP) from the non-affected primary motor cortex (M1) representing the APB area consistently. Resting motor threshold was determined by systemically reducing the stimulus intensity over M1. Motor threshold is defined as the lowest intensity level required to induce MEP with peak-to-peak amplitude of at least $50 \mu \mathrm{V}$ in 5 out of 10 consecutive trials [23]. rTMS was then applied at $1 \mathrm{~Hz}$ at $90 \%$ of MT to the non-lesioned hemisphere extensor digitorum communis $\mathrm{M} 1$ for 20 minutes. $t D C S$

tDCS was administered via Soterix medical $1 \times 1$ tDCS. A saline-soaked cathodal electrode $(5 \mathrm{~cm} \times 7 \mathrm{~cm})$ was applied on the scalp overlying the unaffected motor cortex, while the anodal electrode was placed on the contralateral forehead above the orbit. The current used was 1.5 $\mathrm{mA}$, and the duration of stimulation was 20 minutes.

Each subject would then undergo upper limb rehabilitation training immediately following a NIBS session as part of the routine care. Each subject would receive tailored exercises involving sensorimotor integration, functional muscle activation, and task-dependent re-learning program by an occupational therapist for at least $60 \mathrm{~min}$ utes. Non-invasive electrical brain stimulation and upper limb training were performed three times per week for 2 weeks.

\section{Statistical analyses}

The t-test and Mann-Whitney U test were used to compare clinical demographics between the two intervention groups for continuous data, and chi-square test for categorical data, respectively. Within-group changes at the end of weeks 2 and 4 of training were presented as changes from baseline. Longitudinal data on the motor assessment scale were analyzed by repeated-measures ANOVA for between-group differences. A p-value $<0.05$ was considered as statistically significant.

\section{RESULTS}

A total of 360 patients were admitted for stroke rehabilitation from September 1, 2018 to March 31, 2020. Fifty-one subjects were recruited. Three of them ( 1 in the rTMS group and 2 in the tDCS group) did not complete the program due to changes in disease condition and were transferred to the acute hospital for further care. Fig. 1 shows a consort flow diagram for patient recruitment. The mean age was $64.4 \pm 11.6$ years, and $26(51 \%)$ were male. Thirty-seven patients $(72.5 \%)$ had ischemic stroke, and 14 (27.5\%) had hemorrhagic stroke. Twentynine patients (59.9\%) had left-sided cerebral involvement. For the etiologies of ischemic stroke, there were 30 (78.9\%) small vessel disease, 4 (10.5\%) large vessel disease, 1 (2.6\%) cardioembolic stroke, and 3 (7.9\%) were undetermined. The Oxfordshire classification for infarct location identified 22 (57.9\%) lacunar infarcts, 8 (21.1\%) 


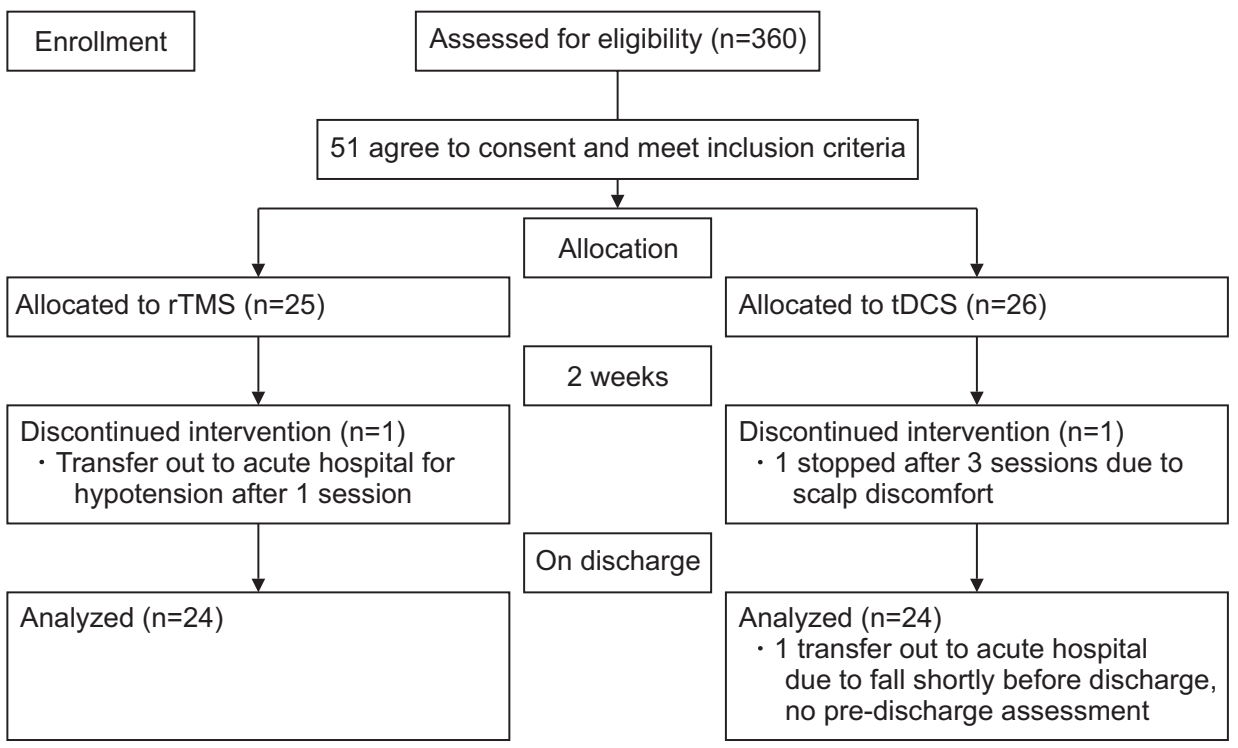

Fig. 1. The consort flow diagram. rTMS, repetitive transcranial magnetic stimulation; tDCS, transcranial direct current stimulation.

Table 1. Comparison of baseline clinical and demographic characteristics

\begin{tabular}{|c|c|c|c|}
\hline & rTMS $(n=25)$ & tDCS $(n=26)$ & p-value \\
\hline Age (yr) & $63.2 \pm 12.8$ & $66.5 \pm 10.1$ & 0.197 \\
\hline Sex, male & $10(40)$ & $15(57.6)$ & 0.206 \\
\hline Stroke side (left hemisphere) & $17(68)$ & $12(46)$ & 0.115 \\
\hline Ischemic stroke & $19(76)$ & $18(69)$ & 0.586 \\
\hline Time from stroke onset to intervention (day) & 18 & 16 & 0.727 \\
\hline \multicolumn{4}{|l|}{ TOAST classification of stroke subtype } \\
\hline Large vessel $(n=4)$ & $3(75)$ & $1(25)$ & \\
\hline Cardioembolic $(\mathrm{n}=1)$ & $1(100)$ & 0 & \\
\hline Small vessel $(n=30)$ & $15(50)$ & $15(50)$ & \\
\hline Undetermined $(\mathrm{n}=3)$ & $1(33)$ & $2(66)$ & 0.506 \\
\hline \multicolumn{4}{|l|}{ Oxfordshire classification of stroke etiology } \\
\hline Lacunar $(\mathrm{n}=22)$ & $10(45.5)$ & $12(54.4)$ & \\
\hline Total anterior circulation $(n=3)$ & $3(100)$ & $0(0)$ & \\
\hline Partial anterior circulation $(n=8)$ & $4(50)$ & $4(50)$ & \\
\hline Posterior circulation $(n=5)$ & $2(40)$ & $3(60)$ & 0.336 \\
\hline Motor Assessment Scale & 45 & 34.5 & 0.196 \\
\hline Modified Ashworth Scale & 0 & 0 & 0.185 \\
\hline Fugl-Meyer arm score test & 55 & 54 & 0.109 \\
\hline Nine Hole Peg Test & 0.18 & 0.05 & 0.193 \\
\hline Modified Barthel Index & 79 & 54 & 0.050 \\
\hline Length of hospitalization (day) & 29 & 28.5 & 0.547 \\
\hline
\end{tabular}

Values are presented as mean \pm standard deviation or number (\%) or median.

rTMS, repetitive transcranial magnetic stimulation; tDCS, transcranial direct current stimulation; TOAST, Trial of Org 10172 in Acute Stroke Treatment.

partial anterior circulation infarcts, $3(7.9 \%)$ total anterior circulation infarcts, and $5(13.2 \%)$ posterior circulation infarcts.
Among these 51 subjects, 25 were randomized to rTMS, and 26 were randomized to tDCS.

Table 1 shows the baseline demographics of the inter- 
(A)

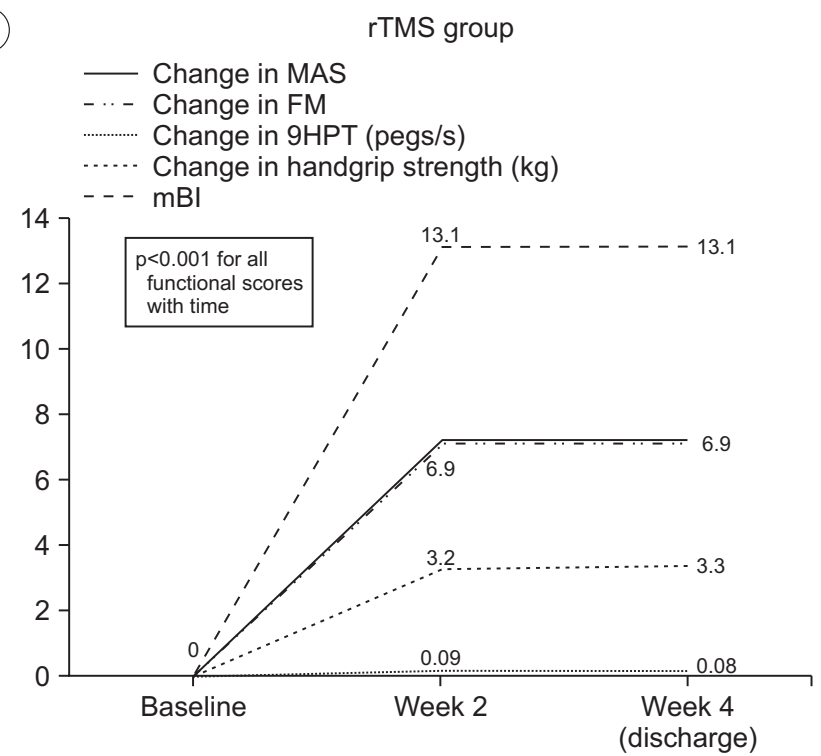

(B)

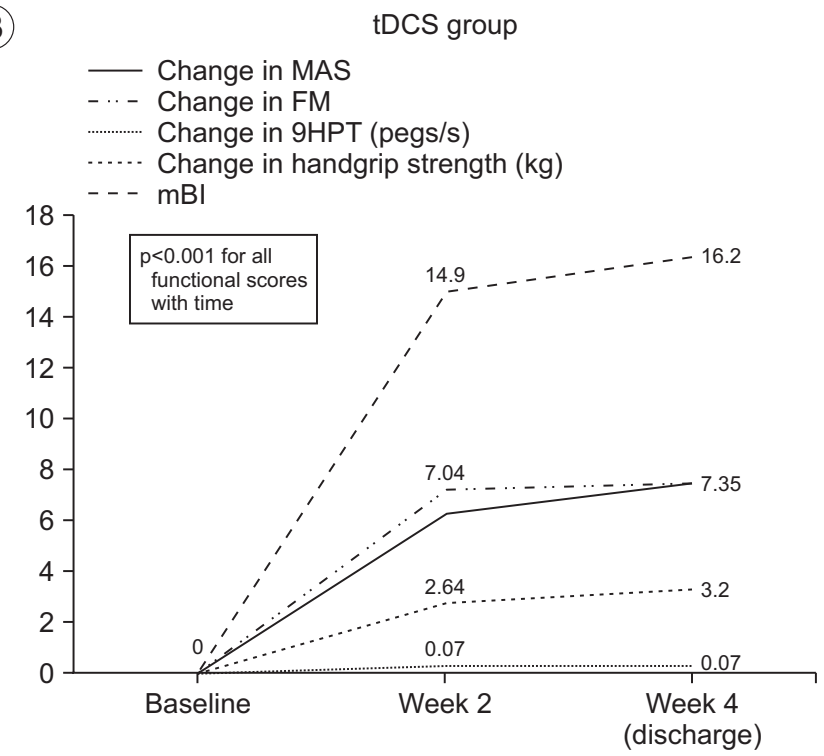

Fig. 2. The within group changes in functional outcome: (A) rTMS group and (B) tDCS group. Each line represents functional outcome scores. rTMS, repetitive transcranial magnetic stimulation; tDCS, transcranial direct current stimulation; MAS, Motor Assessment Scale; FM, Fugl-Meyer arm score test; 9HPT, Nine-Hole Peg Test; mBI, modified Barthel Index.

vention groups. There were no significant differences between the two groups in terms of baseline characteristics. Fig. 2 shows the changes in functional outcomes. Both groups showed a statistically significant improvement in the motor function scores with p-value $<0.001$ for all within-group comparisons with time, except for AS, which did not show any change with time. However, there was no significant difference between tDCS and rTMS on any of the motor function scales (Fig. 3). When we considered non-inferiority of tDCS over rTMS as a $<10 \%$ above the lower boundary of the $95 \%$ confidence interval of rTMS, it was found that for the change in $\mathrm{mBI}$ at weeks 2 and 4 , tDCS yields a $4 \%$ above the lower boundary of the $95 \%$ confidence interval of rTMS, while for the other motor assessments, this difference of confidence interval does not meet. These findings suggest that tDCS was non-inferior for gross physical function as reflected in the $\mathrm{mBI}$, while rTMS may be superior to tDCS on fine hand motor function recovery.

\section{DISCUSSION}

This study aimed to determine the effects of two different stimulation conditions on upper limb motor recovery among patients with recent stroke. Our results show several important findings. First, there was a significant improvement in each of the outcome variables on motor recovery in both intervention groups. The percentage change in the 9HPT, which measured fine hand function, was better in the rTMS group, while those that measured gross motor function, such as $\mathrm{mBI}$, were better in the tDCS group, although the difference was not statistically significant. This inconsistency of motor improvement has been reported in previous studies [24]. Cathodal tDCS and cTBS (continuous theta burst stimulation) produced specific changes in markers of neuroplasticity, although these neural effects cannot be translated into an improvement in motor recovery [25].

There are different mechanisms of rTMS and tDCS on the modulation of interhemispheric driving [25]. tDCS produced a weak polarization of a larger number of neurons, which in turn modulates the ongoing synaptic activity during motor activation [26]. In contrast, rTMS can induce a more focal electrical field. It then generates action potentials in a specific neural circuit [27]. This is more favorable for interventions that involve stimulating specific white matter tracts. It is speculated that rTMS may preferentially affect transcallosal neurons than tDCS [25].

In this study, the beneficial effect of rTMS over non- 

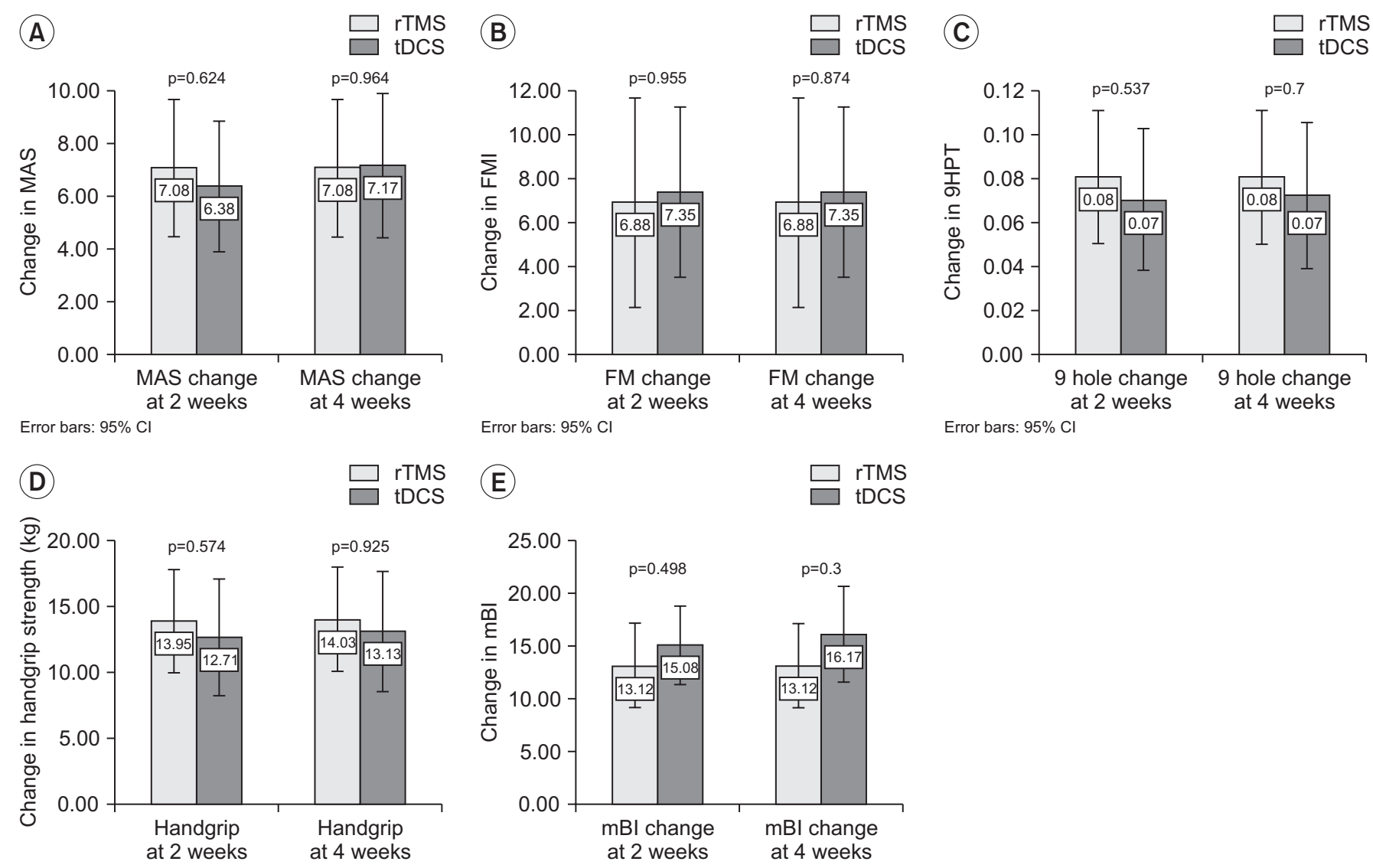

Error bars: $95 \% \mathrm{Cl}$

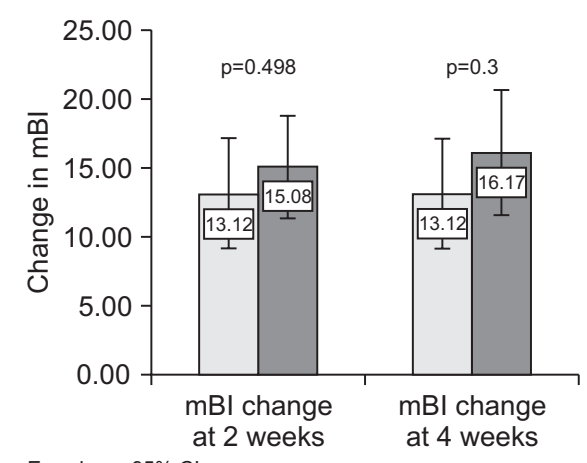

Error bars: $95 \% \mathrm{Cl}$

Error bars: $95 \% \mathrm{Cl}$

Fig. 3. The between group differences in functional outcome. Changes in (A) MAS, (B) FM, (C) 9HPT, (D) handgrip strength, and (E) $\mathrm{mBI}$ from baseline. Each bar and 95\% confidence interval (CI) represent the changes in functional scores in the rTMS group and tDCS group. rTMS, repetitive transcranial magnetic stimulation; tDCS, transcranial direct current stimulation; MAS, Motor Assessment Scale; FM, Fugl-Meyer arm score test; 9HPT, Nine-Hole Peg Test; mBI, modified Barthel Index.

lesional M1 was more robust for fine hand movement than for large motor function. This is in accordance with a pilot study in Thailand [28], who found that object size can influence task difficulty. Small objects require greater precision of the upper limb, which will lead to greater engagement of the cortico-subcortical networks compared to larger objects. This is further supported by a previous study that showed that during complex task execution, there is more involvement of $\mathrm{Ml}$ contralateral to the performing hand [29]. It has been proposed that during complex movement of the paralyzed limb, there is a higher interhemispheric interaction from the nonaffected hemisphere, which may inhibit the engagement of the lesioned M1 [30].

After the intervention, there were improvements in all of the assessment scales in both intervention groups, which persisted for up to 4 weeks: around the time of dis- charge. Several studies have reported an additive effect on motor function immediately following a short course of NIBS and physical therapy when compared with physical therapy alone [31]. The long rehabilitation period and brain stimulation intervention in our study is likely to contribute to a greater overall practice effect on motor function recovery, independent of the type of brain stimulation offered. Furthermore, the additive effect of NIBS on rehabilitation may be important for maintaining newly regained motor skills in our patients with recent stroke. It has been demonstrated that bihemispheric tDCS administered in 10 sessions over 2 weeks with CIMT (carotid intima media thickness) modulated inhibitory networks within interhemispheric pathways, which is essential for motor learning after stroke [32]. This is further supported by another study [8] that tDCS can modulate plasticity within the ipsilesional and non-lesioned corti- 
cospinal pathways by the preferential effects of bihemispheric stimulation. This can lead to the maintenance of functional improvement.

Our finding of NIBS to non-lesioned M1 improves coordination and kinetics as shown by the within-group gain in MAS, 9HPT, and handgrip strength. It appears that rTMS produces better performance in fine hand function than tDCS. It is postulated that over activity within the non-lesioned M1 is maladaptive and may retard the performance of the paralytic hand [28]. Application of rTMS to M1 has been demonstrated by various studies [28] as an improvement in the kinematics of grasp. Downregulation of excitability of the non-lesioned hemisphere after rTMS is considered a potential mechanism that helps to restore balanced interhemispheric inhibition [33]. This downregulation of the non-lesioned M1 leads to a reduction of the interhemispheric inhibition of the extensor digitorum communis representational area in the ipsilesional M1, which will improve the kinematics of hand grasp. The disinhibition of the lesioned MI with rTMS over the non-lesioned M1 may expose its role within the neuronal network that is essential for coordination of the affected hand [28]. Neurophysiological studies [34] have proved that the transformation between kinematics and dynamics is part of the neuronal network that involves high-level planning by M1. This leads to the transformation between the extrinsic representation and intrinsic representation of limb motor behavior. It appears that a certain part of high-level neuronal operations of hand reach and grasp action was inherent in M1, which includes coordination and transformation of visual information into action. Thus, when abnormally high interhemispheric inhibition is released, this lesioned M1 contributes more to the part of the neuronal network that coordinates the reach and grasp components [28].

Further research revealed that changes in one component of motor hand action will alter the movement characteristics of the other component [35]. Therefore, the improvement in hand opening after rTMS may lead to an improvement in the coordination between reach and grasp. This improvement in coordination with the reduction in movement time after rTMS, as evidenced by a better 9HPT, suggested a more efficient performance of the paretic hand when non-lesioned M1 is suppressed.

Some limitations exist in our study. The lack of significant clinical differences between the two groups could be due to the small sample size. Based on the effect size observed in our study, taking the best improvement in 9HPT, approximately 750 subjects would be required for each arm to detect non-inferiority. This large target sample is difficult to achieve in a public setting with limited resources.

We did not have a control group with no intervention. We cannot assess whether the improvement in motor function is a result of natural recovery from minor stroke. As discussed before, due to resource constraints, it is difficult to recruit an adequate number of stroke subjects for a three-group comparison.

We have a heterogeneous sample of strokes with different lesion involvement. There may be inter-individual variability in the response to NIBS. Studies have found that the thickness of the skull and sulcal depth [36], and the genotype of the brain-derived neurotrophic factor [37] can influence the response to tDCS, but does not influence the response to rTMS. Therefore, it is speculated that rTMS may be subject to less variability and may be a more preferable stimulation method.

The subjects in our current study had relatively mild impairment, as shown by a median AS score of 0 and FM score $>50$ out of 66 . We cannot extrapolate our findings to patients with more severe stroke. One can imagine that the improvement in hand performance may be attributed to the learning effect by repeated performance of the same task during the experiment. There is evidence to show that even with short-term practice using the paretic hand can produce improvements in certain aspects of task performance [28]. In our study, each of the tasks was assessed three times only at baseline and at 2 and 4 weeks. This small number of repetitions may not have contributed to restoring the normal interhemispheric balance.

The recruitment rate was not satisfactory. Due to the limited availability of rTMS machines, with our center having only 1 rTMS machine, matching of subjects on TMS and tDCS created constraints on the trial period. This limitation of rTMS could only allow 1 subject to undergo training during the two intervention periods. Furthermore, the eligibility of subjects was mainly affected by the stringent criteria in which either the patient's upper limb function was too poor or too good to be included in the study.

On the other hand, our study provides some insight into 
the recovery of upper limb motor performance among patients with recent stroke. Many studies have reported a significant after-effect of tDCS and rTMS on MEP improvement. The beneficial effect is mostly reflected by an improvement in electrical activity, such as an MEP increment, alone [38]. In clinical practice, it will be preferable if the treatment effect of the specific intervention can be translated in terms of clinical responsiveness rather than electrical activity. Studies have demonstrated that these two interventions work well in patients with acute stroke. Recent studies have demonstrated that rTMS can facilitate motor recovery in patients with acute stroke when compared with sham intervention [39]. A more recent study [40] showed that there were statistical and clinical improvements after tDCS in motor function and somatosensory functions compared with control.

This study is the first in our locality to detect the effects of two NIBS methods on upper limb motor recovery. We were able to show a significant improvement in both intervention methods for motor recovery. This may suggest that these two NIBS methods can augment further recovery in addition to conventional rehabilitation.

In conclusion, both tDCS and rTMS can improve upper limb motor recovery in patients with recent stroke. A larger clinical trial is warranted to confirm which NIBS method can produce more favorable motor recovery in patients with stroke.

\section{CONFLICT OF INTEREST}

No potential conflict of interest relevant to this article was reported.

\section{ACKNOWLEDGMENTS}

This study was supported by the Tung Wah Group of Hospitals Research Fund.

\section{AUTHOR CONTRIBUTION}

Conceptualization: Miu KD, Kok C. Methodology: Miu KD, Kok C, Leung SS, Chan E, Wong E. Formal analysis: Miu KD. Funding acquisition: Miu KD. Project administration: Miu KD. Writing - original draft: Miu KD. Writing - rview and editing: Miu KD, Kok C. Approval of final manuscript: all authors.

\section{REFERENCES}

1. Nichols-Larsen DS, Clark PC, Zeringue A, Greenspan A, Blanton S. Factors influencing stroke survivors' quality of life during subacute recovery. Stroke 2005;36:1480-4.

2. Broeks JG, Lankhorst GJ, Rumping K, Prevo AJ. The long-term outcome of arm function after stroke: results of a follow-up study. Disabil Rehabil 1999;21:35764.

3. Kwakkel G, Kollen BJ, van der Grond J, Prevo AJ. Probability of regaining dexterity in the flaccid upper limb: impact of severity of paresis and time since onset in acute stroke. Stroke 2003;34:2181-6.

4. Luke C, Dodd KJ, Brock K. Outcomes of the Bobath concept on upper limb recovery following stroke. Clin Rehabil 2004;18:888-98.

5. Kwakkel G, Wagenaar RC, Koelman TW, Lankhorst GJ, Koetsier JC. Effects of intensity of rehabilitation after stroke: a research synthesis. Stroke 1997;28:1550-6.

6. Langhorne P, Wagenaar R, Partridge C. Physiotherapy after stroke: more is better? Physiother Res Int 1996;1:75-88.

7. Mansur CG, Fregni F, Boggio PS, Riberto M, GallucciNeto J, Santos CM, et al. A sham stimulation-controlled trial of rTMS of the unaffected hemisphere in stroke patients. Neurology 2005;64:1802-4.

8. Goodwill AM, Teo WP, Morgan P, Daly RM, Kidgell DJ. Bihemispheric-tDCS and upper limb rehabilitation improves retention of motor function in chronic stroke: a pilot study. Front Hum Neurosci 2016;10:258.

9. Hallett M. Transcranial magnetic stimulation: a primer. Neuron 2007;55:187-99.

10. Di Pino G, Pellegrino G, Assenza G, Capone F, Ferreri F, Formica D, et al. Modulation of brain plasticity in stroke: a novel model for neurorehabilitation. Nat Rev Neurol 2014;10:597-608.

11. Nowak DA, Grefkes C, Ameli M, Fink GR. Interhemispheric competition after stroke: brain stimulation to enhance recovery of function of the affected hand. Neurorehabil Neural Repair 2009;23:641-56.

12. Fregni F, Boggio PS, Valle AC, Rocha RR, Duarte J, Ferreira MJ, et al. A sham-controlled trial of a 5-day course of repetitive transcranial magnetic stimulation of the unaffected hemisphere in stroke patients. Stroke 2006;37:2115-22. 
13. Liepert J, Zittel S, Weiller C. Improvement of dexterity by single session low-frequency repetitive transcranial magnetic stimulation over the contralesional motor cortex in acute stroke: a double-blind placebocontrolled crossover trial. Restor Neurol Neurosci 2007;25:461-5.

14. Nitsche MA, Cohen LG, Wassermann EM, Priori A, Lang N, Antal A, et al. Transcranial direct current stimulation: state of the art 2008. Brain Stimul 2008;1:206-23.

15. Bastani A, Jaberzadeh S. Does anodal transcranial direct current stimulation enhance excitability of the motor cortex and motor function in healthy individuals and subjects with stroke: a systematic review and meta-analysis. Clin Neurophysiol 2012;123:644-57.

16. Takeuchi N, Izumi S. Noninvasive brain stimulation for motor recovery after stroke: mechanisms and future views. Stroke Res Treat 2012;2012:584727.

17. Bamford J, Sandercock P, Dennis M, Burn J, Warlow C. Classification and natural history of clinically identifiable subtypes of cerebral infarction. Lancet 1991;337:1521-6.

18. Adams Jr HP, Bendixen BH, Kappelle LJ, Biller J, Love $\mathrm{BB}$, Gordon DL, et al. Classification of subtype of acute ischemic stroke: definitions for use in a multicenter clinical trial TOAST (Trial of Org 10172 in Acute Stroke Treatment). Stroke 1993;24:35-41.

19. Carr JH, Shepherd RB, Nordholm L, Lynne D. Investigation of a new motor assessment scale for stroke patients. Phys Ther 1985;65:175-80.

20. Ansari NN, Naghdi S, Arab TK, Jalaie S. The interrater and intrarater reliability of the Modified Ashworth Scale in the assessment of muscle spasticity: limb and muscle group effect. NeuroRehabilitation 2008;23:231-7.

21. Duncan PW, Propst M, Nelson SG. Reliability of the Fugl-Meyer assessment of sensorimotor recovery following cerebrovascular accident. Phys Ther 1983;63:1606-10.

22. Oxford Grice K, Vogel KA, Le V, Mitchell A, Muniz S, Vollmer MA. Adult norms for a commercially available Nine Hole Peg Test for finger dexterity. Am J Occup Ther 2003;57:570-3.

23. Maeda F, Keenan JP, Tormos JM, Topka H, PascualLeone A. Modulation of corticospinal excitability by repetitive transcranial magnetic stimulation. Clin
Neurophysiol 2000;111:800-5.

24. Avenanti A, Coccia M, Ladavas E, Provinciali L, Ceravolo MG. Low-frequency rTMS promotes use-dependent motor plasticity in chronic stroke: a randomized trial. Neurology 2012;78:256-64.

25. Nicolo P, Magnin C, Pedrazzini E, Plomp G, Mottaz A, Schnider A, et al. Comparison of neuroplastic responses to cathodal transcranial direct current stimulation and continuous theta burst stimulation in subacute stroke. Arch Phys Med Rehabil 2018;99:862-72.

26. Bikson M, Name A, Rahman A. Origins of specificity during tDCS: anatomical, activity-selective, and inputbias mechanisms. Front Hum Neurosci 2013;7:688.

27. Di Lazzaro V, Rothwell JC. Corticospinal activity evoked and modulated by non-invasive stimulation of the intact human motor cortex. J Physiol 2014;592:4115-28.

28. Tretriluxana J, Kantak S, Tretriluxana S, Wu AD, Fisher BE. Improvement in paretic arm reach-to-grasp following low frequency repetitive transcranial magnetic stimulation depends on object size: a pilot study. Stroke Res Treat 2015;2015:498169.

29. Gerloff C, Corwell B, Chen R, Hallett M, Cohen LG. The role of the human motor cortex in the control of complex and simple finger movement sequences. Brain 1998;121:1695-709.

30. Murase N, Duque J, Mazzocchio R, Cohen LG. Influence of interhemispheric interactions on motor function in chronic stroke. Ann Neurol 2004;55:400-9.

31. Lefebvre S, Dricot L, Laloux P, Gradkowski W, Desfontaines P, Evrard F, et al. Neural substrates underlying stimulation-enhanced motor skill learning after stroke. Brain 2015;138:149-63.

32. Bolognini N, Vallar G, Casati C, Latif LA, El-Nazer R, Williams J, et al. Neurophysiological and behavioral effects of tDCS combined with constraint-induced movement therapy in poststroke patients. Neurorehabil Neural Repair 2011;25:819-29.

33. Takeuchi N, Chuma T, Matsuo Y, Watanabe I, Ikoma K. Repetitive transcranial magnetic stimulation of contralesional primary motor cortex improves hand function after stroke. Stroke 2005;36:2681-6.

34. Sergio LE, Hamel-Paquet C, Kalaska JF. Motor cortex neural correlates of output kinematics and kinetics during isometric-force and arm-reaching tasks. J Neurophysiol 2005;94:2353-78. 
35. Haggard P, Wing A. Coordination of hand aperture with the spatial path of hand transport. Exp Brain Res 1998;118:286-92.

36. Opitz A, Paulus W, Will S, Antunes A, Thielscher A. Determinants of the electric field during transcranial direct current stimulation. Neuroimage 2015;109:14050 .

37. Antal A, Chaieb L, Moliadze V, Monte-Silva K, Poreisz $\mathrm{C}$, Thirugnanasambandam $\mathrm{N}$, et al. Brain-derived neurotrophic factor (BDNF) gene polymorphisms shape cortical plasticity in humans. Brain Stimul 2010;3:230-7.

38. Inukai Y, Saito K, Sasaki R, Tsuiki S, Miyaguchi S, Ko- jima S, et al. Comparison of three non-invasive transcranial electrical stimulation methods for increasing cortical excitability. Front Hum Neurosci 2016;10:668.

39. Long H, Wang H, Zhao C, Duan Q, Feng F, Hui N, et al. Effects of combining high- and low-frequency repetitive transcranial magnetic stimulation on upper limb hemiparesis in the early phase of stroke. Restor Neurol Neurosci 2018;36:21-30.

40. Bornheim S, Croisier JL, Maquet P, Kaux JF. Transcranial direct current stimulation associated with physical-therapy in acute stroke patients: a randomized, triple blind, sham-controlled study. Brain Stimul 2020;13:329-36. 